\title{
Exact quark-mass dependence of the Higgs-photon form factor at three loops in QCD
}

\author{
Marco Niggetiedt \\ Institut für Theoretische Teilchenphysik und Kosmologie, RWTH Aachen University, \\ Aachen D-52056, Germany \\ E-mail: marco.niggetiedt@rwth-aachen.de
}

\begin{abstract}
We follow up on our discussion of the exact quark-mass dependence of the Higgs-gluon form factor at three loops in QCD [1] and turn our attention to the closely related Higgs-photon form factor. Similarly to our previous work, we intend to examine the form factor for the decay of a Higgs-boson with variable mass into two photons at the three-loop level in QCD. The set of master integrals is known numerically due to prior work on the Higgs-gluon form factor and is exploited to obtain expansions around the threshold as well as in the high-energy limit. Our results may be utilised to derive the photonic decay rate of the Higgs-boson through next-to-next-to-leading order.
\end{abstract}

Keywords: Higgs Physics, Perturbative QCD

ARXIV EPRINT: 2009.10556 


\section{Contents}

1 Introduction 1

2 Definitions 2

3 Results 5

4 Conclusions and outlook $\quad 6$

$\begin{array}{ll}\text { A Large-mass expansion } & 9\end{array}$

$\begin{array}{ll}\text { B Threshold expansion } & 13\end{array}$

$\begin{array}{ll}\text { C High-energy expansion } & 16\end{array}$

$\begin{array}{ll}\text { D Supplemental material } & 17\end{array}$

\section{Introduction}

Since its discovery in 2012 at the Large Hadron Collider (LHC) by the two collaborations ATLAS and CMS [2,3], the Higgs-boson became one of the most promising candidates to study the Standard Model (SM) and physics beyond the SM. Even though the SM passed the most precise tests until now, small deviations between theoretical computations and experimental data could reveal missing pieces of a more complete theory of particle physics. It is therefore necessary to investigate the production and decay modes of the Higgs-boson in great detail. According to theory predictions for the branching-ratios (BR) ref. [4], the decay of a $125 \mathrm{GeV}$ Higgs-boson into a pair of bottom-quarks is favoured, but less significant for experimental studies due to the large background at hadron colliders. Despite the fact that the BR for the decay $H \rightarrow \gamma \gamma$ is of $\mathcal{O}\left(10^{-3}\right)$, Higgs-boson decay into a pair of photons belongs to the most relevant decay channels due to the high precision to which the final state particles can be measured.

Moreover, the feature that $H \rightarrow \gamma \gamma$ is a loop induced process makes it an appealing channel to determine not only the Higgs-boson mass with excellent resolution, but also to extract Yukawa couplings, since the Higgs-boson couples to all massive particles running in the loops.

Although the process at hand is loop induced and therefore hard to examine within the framework of a multi-loop calculation, the two-loop corrections to the Higgs-decay were computed a long time ago in the heavy-top limit in refs. [5, 6] and, subsequently, results covering the region below and even above the top-threshold followed with refs. [710] via numerical integration. A decade later, these results became available in analytical 
form [11-13]. However, the three-loop calculation seems to be more involved. Nevertheless, expansions in the regime, where the mass of the mediating quark is considered much larger than the mass of the Higgs-boson, have been employed to determine the three-loop form factor as a series expansion in terms of the fraction of the mentioned masses $[14,15]$. The only analytical result currently available captures contributions originating from diagrams with one massless fermion loop [16]. Finally, the large logarithms of $\mathcal{O}\left(\alpha \alpha_{s}^{2} L^{k}\right)$ have been predicted in refs. [17-20].

The paper at hand was motivated by the authors of refs. [19, 20], who kindly requested the availability of the Higgs-photon form factor expanded in the high-energy limit to perform consistency-checks with their own results. Since the diagrams that account for the Higgs-photon form factor form a subset of diagrams contributing to the Higgs-gluon form factor, we closely follow our previous publication ref. [1]. Hence, the reduction table for the simplification to master integrals and their numerical solution, which was obtained via solving a system of differential equations, can be exploited to determine the desired expansions and the form factor itself.

Throughout this publication, we treat the diagrams shown in figure 1 that incorporate two fermion loops as follows: either both fermions are massive quarks or one of them, in particular the one that couples to the Higgs-boson, is massive and the other one massless. In this way, we arrive at the three-loop Higgs-photon form factor in QCD with a single massive quark flavour.

This publication is structured as follows: in the following section, we clarify the notation and conventions used in this paper. Subsequently, we briefly discuss our findings and draw conclusions. Explicit results for the expansions of the missing piece of the three-loop form factor and information on the contents of the supplementary material are given in the appendices. An entire chapter dedicated for a thorough discussion on the technical details is given in ref. [1].

\section{Definitions}

In this section, we introduce the notation and conventions used throughout this paper. The process of interest is the decay of a Higgs-boson with arbitrary mass into two photons with momenta $p_{1}$ and $p_{2}$ and helicities $\lambda_{1}$ and $\lambda_{2}$. We write the amplitude as follows:

$$
-i \mathcal{M}\left[H \rightarrow \gamma\left(p_{1}, \lambda_{1}\right)+\gamma\left(p_{2}, \lambda_{2}\right)\right] \equiv i\left[\left(\epsilon_{1} \cdot p_{2}\right)\left(\epsilon_{2} \cdot p_{1}\right)-\left(\epsilon_{1} \cdot \epsilon_{2}\right)\left(p_{2} \cdot p_{1}\right)\right] \frac{1}{v} \frac{\alpha}{\pi} Q_{q}^{2} \mathcal{C}
$$

$Q_{q}$ denotes the electric charge of the top-quark, $\alpha$ is the electromagnetic coupling constant and $v$ indicates the Vacuum Expectation Value originating from the tree-level Lagrangian term $-M \bar{Q} Q H / v$, which is responsible for the coupling of the quark field to the Higgsboson. For the photon polarisation vectors, the normalisation conditions hold:

$$
\epsilon_{i} \equiv \epsilon\left(\boldsymbol{p}_{i}, \lambda_{i}\right), \quad \epsilon_{i} \cdot p_{i}=0, \quad \epsilon_{i} \cdot \epsilon_{i}^{*}=-1, \quad i=1,2
$$




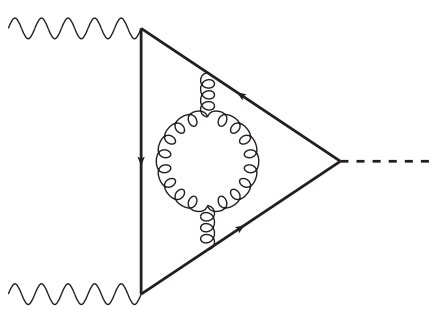

(a)

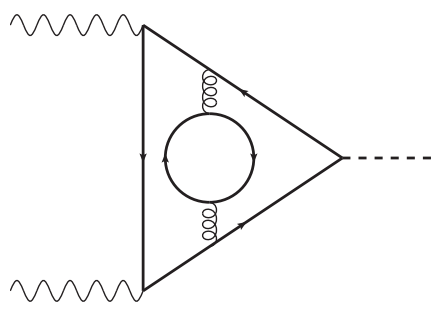

(c)

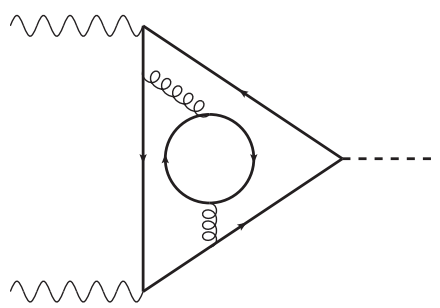

(e)

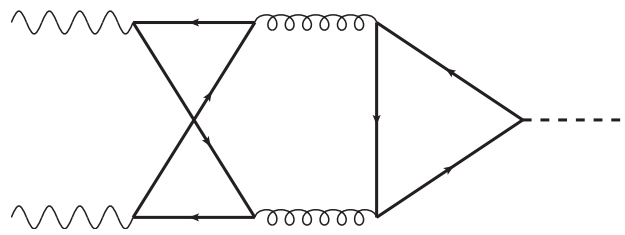

(g)

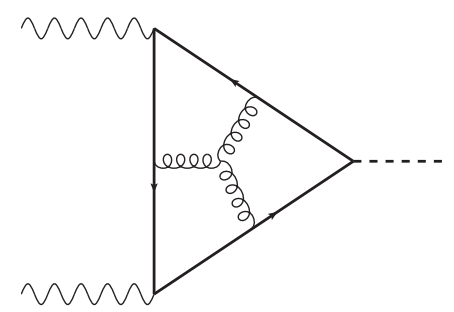

(b)

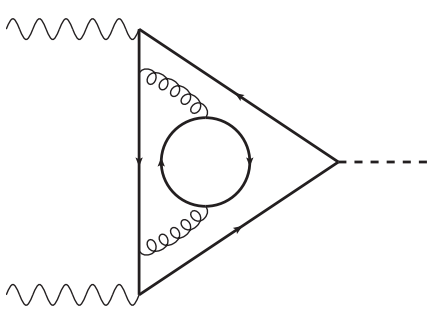

(d)

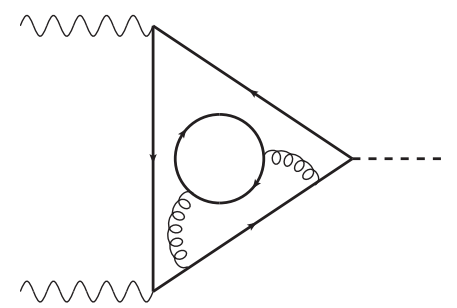

(f)

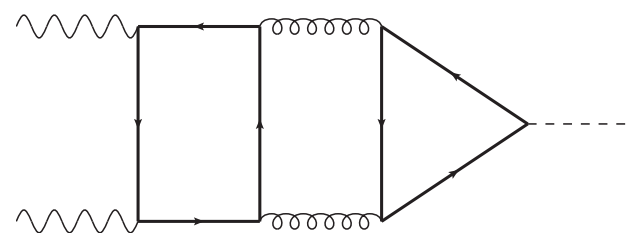

(h)

Figure 1. Sample three-loop Feynman diagrams for the decay of a Higgs-boson into two photons. Diagrams $\mathrm{c}-\mathrm{h}$ visualise all non-vanishing contributions involving two closed fermion loops.

In accordance with eq. (2.1), the Form Factor $\mathcal{C}$ admits a perturbative expansion in terms of the strong coupling constant, $\alpha_{s}$.

$$
\mathcal{C}=\mathcal{C}^{(0)}+\frac{\alpha_{s}}{\pi} \mathcal{C}^{(1)}+\left(\frac{\alpha_{s}}{\pi}\right)^{2} \mathcal{C}^{(2)}+\mathcal{O}\left(\alpha_{s}^{3}\right)
$$

As far as renormalisation is concerned, we stick to the same conventions as in [1] for the sake of convenience. We define the strong coupling constant in $\overline{\mathrm{MS}}$ scheme with massivequark decoupling. The $\beta$-function for $n_{l}$ massless quarks gives rise to the dependence on the renormalisation scale: $\alpha_{s} \equiv \alpha_{s}^{\left(n_{l}\right)}(\mu)$. Furthermore, the quark-mass and henceforth the Yukawa coupling are renormalised in the on-shell scheme. All relevant constants for renormalisation and decoupling can be taken from [21-25]. 
In contrast to the known one- and two-loop contributions, $\mathcal{C}^{(0)}$ and $\mathcal{C}^{(1)}$, respectively, the three-loop coefficient, $\mathcal{C}^{(2)}$, may be subdivided into contributions stemming from different classes of Feynman diagrams:

$$
\mathcal{C}^{(2)}=\mathcal{C}^{(2,0)}+n_{h} \mathcal{C}^{(2,1)}+n_{l} \mathcal{C}^{(2,2)}+\sum_{k=1}^{n_{l}}\left(\frac{Q_{k}}{Q_{q}}\right)^{2} \mathcal{C}^{(2,3)}
$$

Here, the splitting into the four tree-loop coefficients, $\mathcal{C}^{(2, k)}$, is motivated by the fact that Feynman diagrams with more than one fermion loop contribute at three-loop level for the first time. $\mathcal{C}^{(2,0)}$ gathers all diagrams with exactly one closed fermion chain to which the external particles are necessarily attached. Two typical diagrams are shown in figures 1ab. Diagrams that contribute to $\mathcal{C}^{(2,1)}$ are those, which embed two massive fermion loops depicted in figures $1 \mathrm{c}-\mathrm{h}$. We do not distinguish between diagrams in which one of the fermion loops is neither connected to the Higgs-boson nor to the external photons, as well as those where one of the fermion loops couples to the photons and the other one to the Higgsboson. In this context, $n_{h}$ indicates the number of massive quarks not coupling to the Higgsboson. With the three-loop coefficients $\mathcal{C}^{(2,2)}$ and $\mathcal{C}^{(2,3)}$, which are known analytically [16], we associate all Feynman diagrams that involve one massless and one massive fermion loop. One usually differentiates between singlet and non-singlet contributions. Singlet diagrams (figures $1 \mathrm{~g}-\mathrm{h}$ ) collected in $\mathcal{C}^{(2,3)}$ incorporate one massive fermion loop attached to the Higgs-boson and one massless fermion loop that couples to the external photons. Hence, we have to sum over the electric charges of all massless fermion flavours. In contrast to that, the diagrams displayed in figures $1 \mathrm{c}-\mathrm{f}$ with a massless fermion loop in the centre account for the non-singlet part, $\mathcal{C}^{(2,2)} \cdot \mathcal{C}^{(2,0)}$ encompasses non-singlet diagrams only, but as pointed out before, $\mathcal{C}^{(2,1)}$ covers both singlet and non-singlet parts.

The form factors and their individual components depend on the fraction of the masses of the Higgs-boson and the mediating massive quark and on the logarithm containing the renormalisation scale:

$$
\begin{aligned}
\mathcal{C}^{(\cdots)} & \equiv \mathcal{C}^{(\cdots)}\left(z, L_{\mu}\right), \\
z & \equiv \frac{s}{4 M^{2}}+i 0^{+}, \quad L_{\mu} \equiv \ln \left(-\frac{\mu^{2}}{s+i 0^{+}}\right), \quad s \equiv\left(p_{1}+p_{2}\right)^{2} .
\end{aligned}
$$

In order to clarify the notation, we state the leading contribution:

$$
\mathcal{C}^{(0)}=\frac{C_{A}}{T_{F}} \frac{1}{2 z}\left\{1-\left(1-\frac{1}{z}\right)\left[\frac{1}{2} \ln \left(\frac{\sqrt{1-1 / z}-1}{\sqrt{1-1 / z}+1}\right)\right]^{2}\right\},
$$

which in the heavy-top limit takes the value:

$$
\mathcal{C}^{(0)}[z=0]=2 .
$$

The form factor is scale-independent implying

$$
\frac{\mathrm{d} \ln \mathcal{C}}{\mathrm{d} \ln \mu}=0 .
$$


Thus, the dependence of the form factor on the aforementioned logarithm, $L_{\mu}$, can be expressed with the aid of the coefficients of the QCD $\beta$-function:

$$
\begin{aligned}
\mathcal{C}^{(0)} & =\mathcal{C}^{(0)}\left[L_{\mu}=0\right], \\
\mathcal{C}^{(1)} & =\mathcal{C}^{(1)}\left[L_{\mu}=0\right], \\
\mathcal{C}^{(2)} & =\mathcal{C}^{(2)}\left[L_{\mu}=0\right]+\frac{b_{0}}{4} \mathcal{C}^{(1)} L_{\mu} .
\end{aligned}
$$

For the sake of completeness, we quote the first coefficient of the $\beta$-function:

$$
b_{0}=\frac{11}{3} C_{A}-\frac{4}{3} T_{F} n_{l} .
$$

\section{Results}

In this section, we briefly present out findings. The scale-dependence is fixed such that $L_{\mu}=0$ and can easily be restored by applying eqs. (2.10).

We check our results for the light-fermion contributions, in particular, the three-loop coefficients $\mathcal{C}^{(2,2)}$ and $\mathcal{C}^{(2,3)}$, numerically against the analytical results in ref. [16]. For the numerical probes as well as for the expansions in the kinematic limits, we find full agreement.

Similar to our previous work, the exact result for the Higgs-photon form factor at threeloop level, $\mathcal{C}^{(2)}$, is stored in the form of a univariate interpolation based on nearly 200.000 numerical probes distributed over the physical parameter space in the variable $z$. Other than that, we derived high-order large-mass, threshold and high-energy expansions, which cover most parts of the parameter space to sufficient precision. The radii of convergence of the three expansions are limited due to singularities located at $z=0, z=1$ and $1 / z=0$. The supplementary material HaaNiggetiedt.m attached to this paper shipped with this publication contains the large-mass expansion with exact coefficients truncated at $\mathcal{O}\left(z^{100}\right)$, the threshold expansion truncated at $\mathcal{O}\left((1-z)^{20}\right)$ and the high-energy expansion truncated at $\mathcal{O}\left(1 / z^{8}\right)$. The latter ones are expansions with numerical coefficients. We choose the truncation order of the numerical expansions such that we can confidently guarantee the correctness of at least ten digits for every numerical coefficient.

A comparison of the mentioned expansions with the exact numerical result for the sum of three-loop coefficients $\mathcal{C}^{(2,0)}+\mathcal{C}^{(2,1)}$ is illustrated in figure 2. For those values of $z$ which are not covered by expansions, we provide interpolation tables in tables 1 and 2 , where we reshaped the domain of positive $z$ values to the interval $(0,1)$ by applying the conformal mapping

$$
z(\rho) \equiv \frac{4 \rho}{1-\rho}, \quad \rho(z)=\frac{z}{4+z}, \quad \rho \in(0,1) .
$$

With a relative error of at most $10^{-5}$, the exact result for the sum of three-loop coefficients $\mathcal{C}^{(2,0)}+\mathcal{C}^{(2,1)}$ is approximated as follows:

$0<\rho<1 / 6$ - large-mass expansion, appendix A and figure 3;

$1 / 6 \leq \rho<1 / 4 \quad$ - threshold expansion, appendix B and figure 4; 


\begin{tabular}{|c|c|c|c|}
\hline$\rho$ & $\mathcal{C}^{(2,0)}+\mathcal{C}^{(2,1)}$ & $\rho$ & $\mathcal{C}^{(2,0)}+\mathcal{C}^{(2,1)}$ \\
\hline $1 / 4$ & $95.61412709+176.89801445 i$ & $3 / 8$ & $-114.50707747+154.19306610 i$ \\
$51 / 200$ & $83.07596079+184.20923088 i$ & $19 / 50$ & $-118.45992102+149.95386054 i$ \\
$13 / 50$ & $70.66622472+190.11797906 i$ & $77 / 200$ & $-122.15870044+145.68482348 i$ \\
$53 / 200$ & $58.47564245+194.78093076 i$ & $39 / 100$ & $-125.61387922+141.39604695 i$ \\
$27 / 100$ & $46.57137881+198.33693618 i$ & $79 / 200$ & $-128.83557098+137.09659724 i$ \\
$11 / 40$ & $35.00244955+200.90932597 i$ & $2 / 5$ & $-131.83353409+132.79461434 i$ \\
$7 / 25$ & $23.80378890+202.60783914 i$ & $81 / 200$ & $-134.61717010+128.49740137 i$ \\
$57 / 200$ & $12.99935281+203.53026304 i$ & $41 / 100$ & $-137.19552522+124.21150499 i$ \\
$29 / 100$ & $2.60451436+203.76384339 i$ & $83 / 200$ & $-139.57729434+119.94278778 i$ \\
$59 / 200$ & $-7.37206987+203.38650552 i$ & $21 / 50$ & $-141.77082686+115.69649353 i$ \\
$3 / 10$ & $-16.92699443+202.46791772 i$ & $17 / 40$ & $-143.78413407+111.47730602 i$ \\
$61 / 200$ & $-26.06094755+201.07042088 i$ & $43 / 100$ & $-145.62489760+107.28940212 i$ \\
$31 / 100$ & $-34.77779234+199.24984361 i$ & $87 / 200$ & $-147.30047872+103.13649972 i$ \\
$63 / 200$ & $-43.08383477+197.05621891 i$ & $11 / 25$ & $-148.81792823+99.02190109 i$ \\
$8 / 25$ & $-50.98723878+194.53441538 i$ & $89 / 200$ & $-150.18399666+94.94853204 i$ \\
$13 / 40$ & $-58.49755811+191.72469437 i$ & $9 / 20$ & $-151.40514481+90.91897739 i$ \\
$33 / 100$ & $-65.62536110+188.66320244 i$ & $91 / 200$ & $-152.48755434+86.93551306 i$ \\
$67 / 200$ & $-72.38193010+185.38240734 i$ & $23 / 50$ & $-153.43713829+83.00013522 i$ \\
$17 / 50$ & $-78.77902071+181.91148449 i$ & $93 / 200$ & $-154.25955161+79.11458662 i$ \\
$69 / 200$ & $-84.82866943+178.27666003 i$ & $47 / 100$ & $-154.96020144+75.28038056 i$ \\
$7 / 20$ & $-90.54304032+174.50151571 i$ & $19 / 40$ & $-155.54425729+71.49882262 i$ \\
$71 / 200$ & $-95.93430346+170.60726020 i$ & $12 / 25$ & $-156.01666080+67.77103038 i$ \\
$9 / 25$ & $-101.01453909+166.61297072 i$ & $97 / 200$ & $-156.38213534+64.09795135 i$ \\
$73 / 200$ & $-105.79566262+162.53580859 i$ & $49 / 100$ & $-156.64519521+60.48037931 i$ \\
$37 / 100$ & $-110.28936677+158.39121163 i$ & $99 / 200$ & $-156.81015439+56.91896909 i$ \\
\hline
\end{tabular}

Table 1. Numerical values of the three-loop coefficient $\mathcal{C}^{(2,0)}+\mathcal{C}^{(2,1)}$ at $L_{\mu}=0$, for $1 / 4 \leq \rho \equiv$ $z /(4+z)<1 / 2$.
$1 / 4 \leq \rho<3 / 4 \quad$ - interpolation of a sample of numerical values, tables 1 and 2;
$3 / 4 \leq \rho<1 \quad$ - high-energy expansion, appendix $\mathrm{C}$ and figure 5.

\section{Conclusions and outlook}

Provided the findings of this paper, the Higgs-photon form factor is now known exactly at the three-loop level in QCD with a single massive quark-flavour. Moreover, the longing for the desired expansions has been satisfied. We presented the results with the Yukawa 


\begin{tabular}{|c|c|c|c|}
\hline$\rho$ & $\mathcal{C}^{(2,0)}+\mathcal{C}^{(2,1)}$ & $\rho$ & $\mathcal{C}^{(2,0)}+\mathcal{C}^{(2,1)}$ \\
\hline $1 / 2$ & $-156.88113479+53.41425013 i$ & $5 / 8$ & $-137.132840055-15.702661678 i$ \\
$101 / 200$ & $-156.86207296+49.96663944 i$ & $63 / 100$ & $-135.749456670-17.756266155 i$ \\
$51 / 100$ & $-156.75672881+46.57645720 i$ & $127 / 200$ & $-134.335469634-19.758491416 i$ \\
$103 / 200$ & $-156.56869815+43.24393631 i$ & $16 / 25$ & $-132.892081100-21.709726182 i$ \\
$13 / 25$ & $-156.30142058+39.96922935 i$ & $129 / 200$ & $-131.420442234-23.610352498 i$ \\
$21 / 40$ & $-155.95818634+36.75241608 i$ & $13 / 20$ & $-129.921655363-25.460744615 i$ \\
$53 / 100$ & $-155.54214291+33.59351066 i$ & $131 / 200$ & $-128.396776022-27.261267930 i$ \\
$107 / 200$ & $-155.05630130+30.49246836 i$ & $33 / 50$ & $-126.846814904-29.012277977 i$ \\
$27 / 50$ & $-154.50354211+27.44919179 i$ & $133 / 200$ & $-125.272739719-30.714119456 i$ \\
$109 / 200$ & $-153.88662135+24.46353662 i$ & $67 / 100$ & $-123.675476970-32.367125304 i$ \\
$11 / 20$ & $-153.20817593+21.53531684 i$ & $27 / 40$ & $-122.055913644-33.971615786 i$ \\
$111 / 200$ & $-152.47072896+18.66430965 i$ & $17 / 25$ & $-120.414898822-35.527897609 i$ \\
$14 / 25$ & $-151.67669487+15.85025982 i$ & $137 / 200$ & $-118.753245223-37.036263057 i$ \\
$113 / 200$ & $-150.82838414+13.09288386 i$ & $69 / 100$ & $-117.071730670-38.496989120 i$ \\
$57 / 100$ & $-149.92800802+10.39187374 i$ & $139 / 200$ & $-115.371099493-39.910336636 i$ \\
$23 / 40$ & $-148.97768284+7.74690031 i$ & $7 / 10$ & $-113.652063866-41.276549423 i$ \\
$29 / 50$ & $-147.97943427+5.15761652 i$ & $141 / 200$ & $-111.915305089-42.595853392 i$ \\
$117 / 200$ & $-146.93520129+2.62366027 i$ & $71 / 100$ & $-110.161474804-43.868455656 i$ \\
$59 / 100$ & $-145.84684003+0.14465717 i$ & $143 / 200$ & $-108.391196166-45.094543592 i$ \\
$119 / 200$ & $-144.71612740-2.27977708 i$ & $18 / 25$ & $-106.605064963-46.274283888 i$ \\
$3 / 5$ & $-143.54476456-4.65003435 i$ & $29 / 40$ & $-104.803650678-47.407821537 i$ \\
$121 / 200$ & $-142.33438025-6.96651202 i$ & $73 / 100$ & $-102.987497519-48.495278782 i$ \\
$61 / 100$ & $-141.086533913-9.229611000 i$ & $147 / 200$ & $-101.157125397-49.536754007 i$ \\
$123 / 200$ & $-139.802718709-11.439733991 i$ & $37 / 50$ & $-99.313030875-50.532320551 i$ \\
$31 / 50$ & $-138.484364399-13.597283772 i$ & $149 / 200$ & $-97.455688067-51.482025454 i$ \\
$5 / 8$ & $-137.132840055-15.702661678 i$ & $3 / 4$ & $-95.585549514-52.385888101 i$ \\
\hline
\end{tabular}

Table 2. Numerical values of the three-loop coefficient $\mathcal{C}^{(2,0)}+\mathcal{C}^{(2,1)}$ at $L_{\mu}=0$, for $1 / 2 \leq \rho \equiv$ $z /(4+z) \leq 3 / 4$

coupling renormalised in the on-shell scheme, which can be translated to any other scheme due to the fact that the one- and two-loop results are available in analytical form.

We finally note that our results may be utilised to obtain the cross section for Higgsboson production via photon-photon fusion and the photonic decay rate of a Higgs-boson through next-to-next-to-leading order in QCD.

Let us again emphasise that the form factor with the most general quark-mass dependence requires additional elaboration of the diagrams with two closed fermion chains. We postpone this analysis to future publications. 


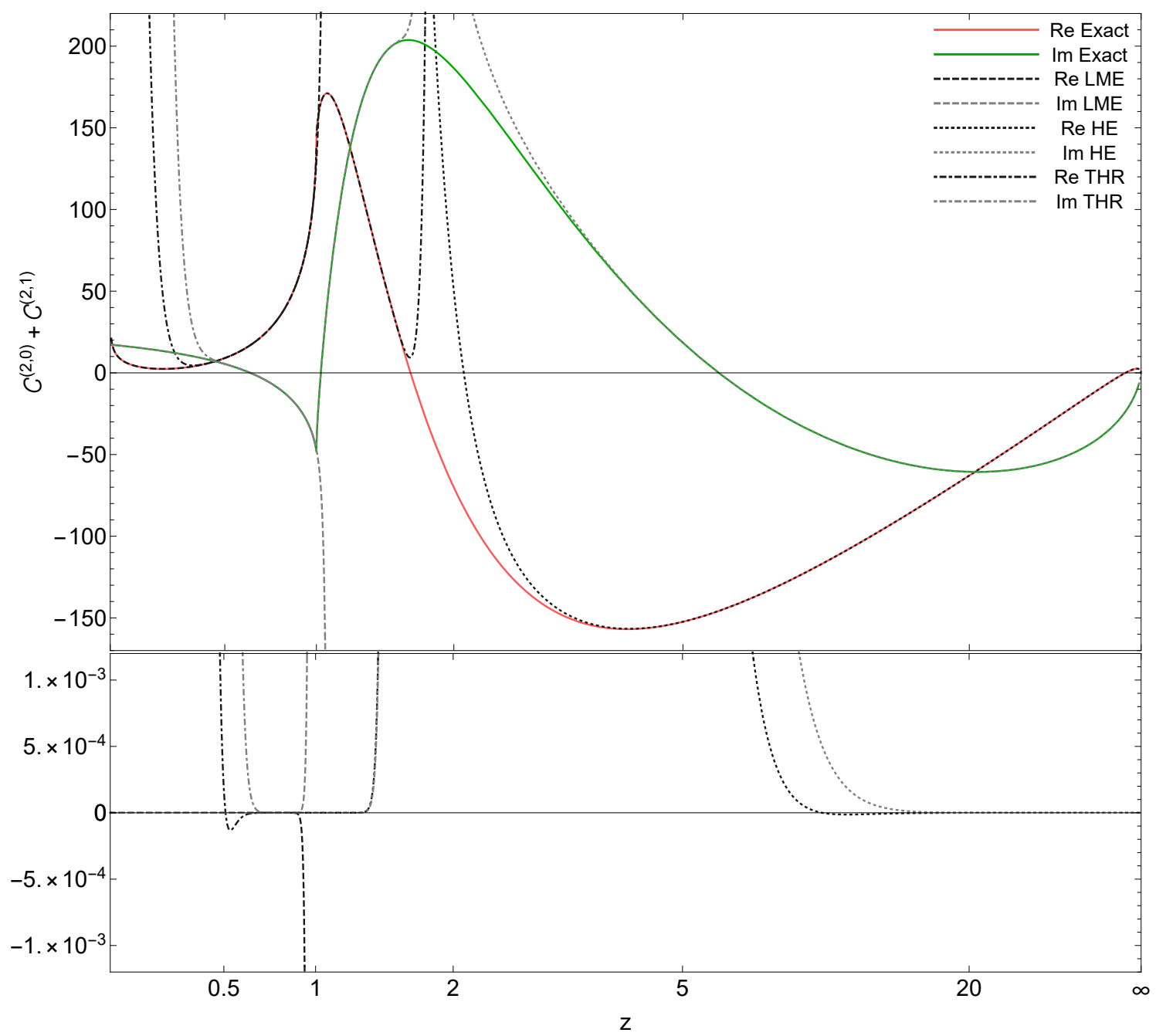

Figure 2. Comparison of the large-mass expansion (LME) up to $\mathcal{O}\left(z^{100}\right)$, threshold expansion (THR) up to $\mathcal{O}\left((1-z)^{20}\right)$ and high-energy expansion (HE) up to $\mathcal{O}\left(1 / z^{8}\right)$ with the sum $\mathcal{C}^{(2,0)}+$ $\mathcal{C}^{(2,1)}$ evaluated numerically $\left(L_{\mu}=0\right)$. The absolute difference between the exact result and the expansions is shown in the bottom panel.

\section{Acknowledgments}

This work was supported by the Deutsche Forschungsgemeinschaft (DFG) under grants 396021762 - TRR 257 and 400140256 - GRK 2497: the physics of the heaviest particles at the Large Hardon Collider. 


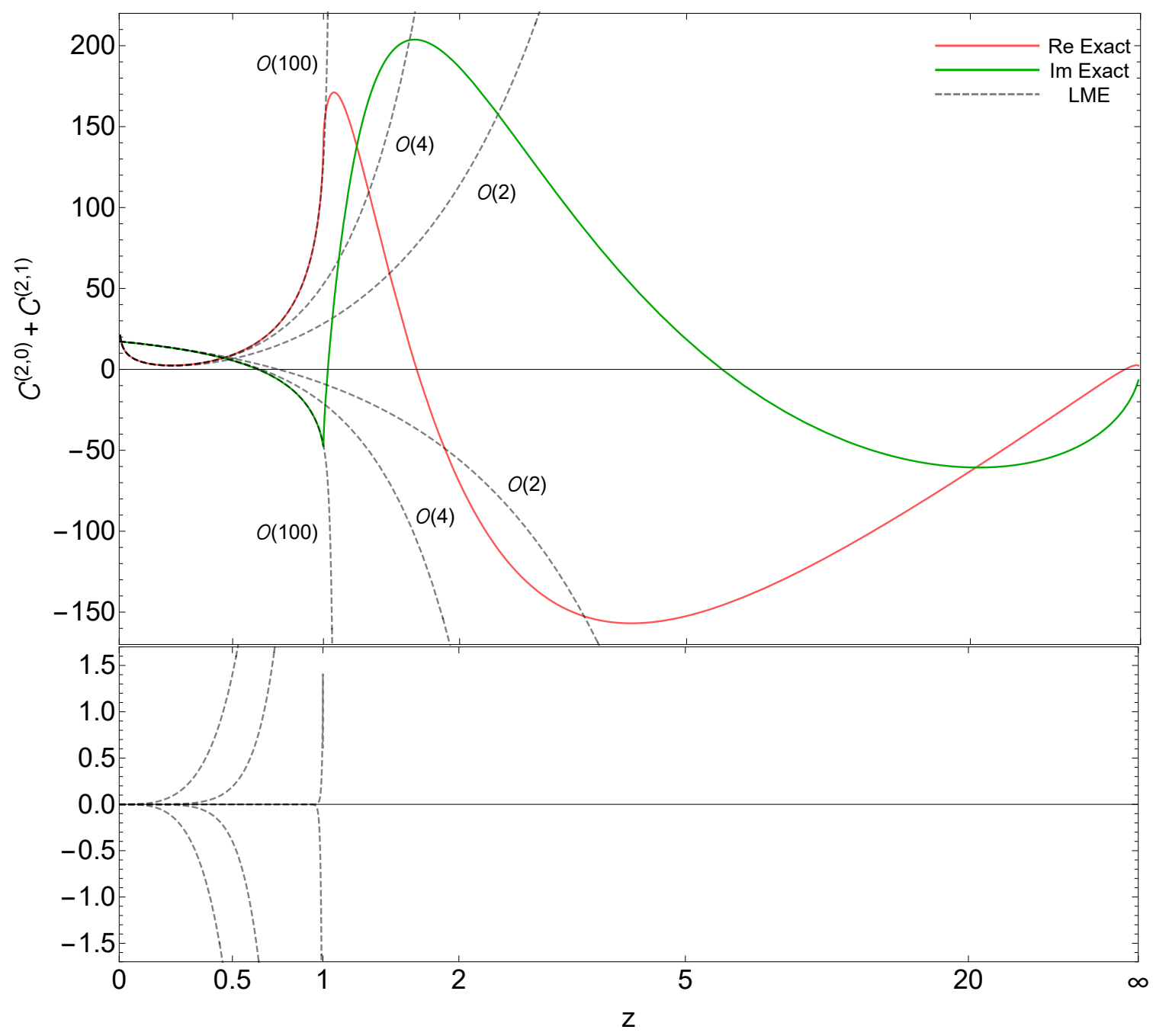

Figure 3. Comparison of the large-mass expansion (LME) up to $\mathcal{O}\left(z^{2}\right), \mathcal{O}\left(z^{4}\right)$ and $\mathcal{O}\left(z^{100}\right)$ with the sum $\mathcal{C}^{(2,0)}+\mathcal{C}^{(2,1)}$ evaluated numerically $\left(L_{\mu}=0\right)$. The absolute difference between the exact result and the expansions is shown in the bottom panel.

\section{A Large-mass expansion}

$$
\begin{aligned}
C^{(2,0)}+C^{(2,1)}= & \sum_{n=0}^{\infty}\left(a_{n, 0}+a_{n, 1} L_{s}\right) z^{n}, \quad L_{s} \equiv \ln \left(-\frac{s}{M^{2}}-i 0^{+}\right) \\
\mathcal{C}^{(2,0)}+\mathcal{C}^{(2,1)}= & -1.777777778-5.500000000 L_{s}+\left(13.11710090+4.970370370 L_{s}\right) z \\
& +\left(13.06368527+3.350405644 L_{s}\right) z^{2}+(10.46030710 \\
& \left.+2.278524565 L_{s}\right) z^{3}+\left(8.320460471+1.610008230 L_{s}\right) z^{4} \\
& +\left(6.703047617+1.188440003 L_{s}\right) z^{5}+(5.493827219
\end{aligned}
$$




$$
\begin{aligned}
& \left.+0.9141819603 L_{s}\right) z^{6}+\left(4.588959115+0.7191908041 L_{s}\right) z^{7} \\
& +\left(3.885458574+0.5838547944 L_{s}\right) z^{8}+(3.340520295 \\
& \left.+0.4795265645 L_{s}\right) z^{9}+\left(2.900355777+0.4036136804 L_{s}\right) z^{10} \\
& +\left(2.548334424+0.3416903422 L_{s}\right) z^{11}+(2.255192075 \\
& \left.+0.2950940542 L_{s}\right) z^{12}+\left(2.014632209+0.2554585379 L_{s}\right) z^{13} \\
& +\left(1.809436750+0.2248978875 L_{s}\right) z^{14}+(1.637551699 \\
& \left.+0.1980436051 L_{s}\right) z^{15}+\left(1.488094722+0.1769594650 L_{s}\right) z^{16} \\
& +\left(1.360807921+0.1579418622 L_{s}\right) z^{17}+(1.248390264 \\
& \left.+0.1428040226 L_{s}\right) z^{18}+\left(1.151340958+0.1288517530 L_{s}\right) z^{19} \\
& +\left(1.064516424+0.1176276537 L_{s}\right) z^{20}+(0.9887120593 \\
& \left.+0.1070919600 L_{s}\right) z^{21}+\left(0.9201563781+0.09854608319 L_{s}\right) z^{22} \\
& +\left(0.8597314175+0.09039720912 L_{s}\right) z^{23}+(0.8045792128 \\
& \left.+0.08374429753 L_{s}\right) z^{24}+\left(0.7555733042+0.07731261247 L_{s}\right) z^{25} \\
& +\left(0.7104879787+0.07203463456 L_{s}\right) z^{26}+(0.6701469601 \\
& \left.+0.06686962473 L_{s}\right) z^{27}+\left(0.6327767878+0.06261386339 L_{s}\right) z^{28} \\
& +\left(0.5991358278+0.05840349687 L_{s}\right) z^{29}+(0.5677833750 \\
& \left.+0.05492318564 L_{s}\right) z^{30}+\left(0.5394092405+0.05144585116 L_{s}\right) z^{31} \\
& +\left(0.5128235733+0.04856423129 L_{s}\right) z^{32}+(0.4886501782 \\
& \left.+0.04565905067 L_{s}\right) z^{33}+\left(0.4658923404+0.04324686803 L_{s}\right) z^{34} \\
& +\left(0.4451128638+0.04079477885 L_{s}\right) z^{35}+(0.4254664886 \\
& \left.+0.03875577657 L_{s}\right) z^{36}+\left(0.4074608421+0.03666714389 L_{s}\right) z^{37} \\
& +\left(0.3903713631+0.03492846512 L_{s}\right) z^{38}+(0.3746563443 \\
& \left.+0.03313477019 L_{s}\right) z^{39}+\left(0.3596887907+0.03164046183 L_{s}\right) z^{40} \\
& +\left(0.3458831478+0.03008860435 L_{s}\right) z^{41}+(0.3326922897
\end{aligned}
$$




$$
\begin{aligned}
& \left.+0.02879512859 L_{s}\right) z^{42}+\left(0.3204917987+0.02744344429 L_{s}\right) z^{43} \\
& +\left(0.3088006713+0.02631651964 L_{s}\right) z^{44}+(0.2979600688 \\
& \left.+0.02513194782 L_{s}\right) z^{45}+\left(0.2875442851+0.02414428832 L_{s}\right) z^{46} \\
& +\left(0.2778639781+0.02310032496 L_{s}\right) z^{47}+(0.2685401083 \\
& \left.+0.02222999757 L_{s}\right) z^{48}+\left(0.2598562662+0.02130519471 L_{s}\right) z^{49} \\
& +\left(0.2514731205+0.02053440900 L_{s}\right) z^{50}+(0.2436501982 \\
& \left.+0.01971125995 L_{s}\right) z^{51}+\left(0.2360822089+0.01902546512 L_{s}\right) z^{52} \\
& +\left(0.2290072400+0.01828956618 L_{s}\right) z^{53}+(0.2221493831 \\
& \left.+0.01767676928 L_{s}\right) z^{54}+\left(0.2157275788+0.01701618233 L_{s}\right) z^{55} \\
& +\left(0.2094914381+0.01646642798 L_{s}\right) z^{56}+(0.2036427601 \\
& \left.+0.01587119071 L_{s}\right) z^{57}+\left(0.1979534480+0.01537616013 L_{s}\right) z^{58} \\
& +\left(0.1926099161+0.01483790614 L_{s}\right) z^{59}+(0.1874036433 \\
& \left.+0.01439060512 L_{s}\right) z^{60}+\left(0.1825072020+0.01390226658 L_{s}\right) z^{61} \\
& +\left(0.1777293453+0.01349678066 L_{s}\right) z^{62}+(0.1732301604 \\
& \left.+0.01305235368 L_{s}\right) z^{63}+\left(0.1688337129+0.01268365449 L_{s}\right) z^{64} \\
& +\left(0.1646888013+0.01227801247 L_{s}\right) z^{65}+(0.1606331223 \\
& \left.+0.01194180331 L_{s}\right) z^{66}+\left(0.1568052431+0.01157054741 L_{s}\right) z^{67} \\
& +\left(0.1530550417+0.01126313939 L_{s}\right) z^{68}+(0.1495117915 \\
& \left.+0.01092247795 L_{s}\right) z^{69}+\left(0.1460362962+0.01064068979 L_{s}\right) z^{70} \\
& +\left(0.1427493678+0.01032734039 L_{s}\right) z^{71}+(0.1395216432 \\
& \left.+0.01006841703 L_{s}\right) z^{72}+\left(0.1364662142+0.009779526526 L_{s}\right) z^{73} \\
& +\left(0.1334625959+0.009541072480 L_{s}\right) z^{74}+(0.1306168216 \\
& \left.+0.009274151328 L_{s}\right) z^{75}+\left(0.1278164469+0.009054075932 L_{s}\right) z^{76} \\
& +\left(0.1251610381+0.008806943918 L_{s}\right) z^{77}+(0.1225454532
\end{aligned}
$$




$$
\begin{aligned}
& \left.+0.008603416011 L_{s}\right) z^{78}+\left(0.1200633223+0.008374157224 L_{s}\right) z^{79} \\
& +\left(0.1176161532+0.008185567502 L_{s}\right) z^{80}+(0.1152921168 \\
& \left.+0.007972492768 L_{s}\right) z^{81}+\left(0.1129987915+0.007797422353 L_{s}\right) z^{82} \\
& +\left(0.1108193188+0.007599037760 L_{s}\right) z^{83}+(0.1086668325 \\
& \left.+0.007436231834 L_{s}\right) z^{84}+\left(0.1066198321+0.007251212232 L_{s}\right) z^{85} \\
& +\left(0.1045965469+0.007099557830 L_{s}\right) z^{86}+(0.1026711854 \\
& \left.+0.006926724446 L_{s}\right) z^{87}+\left(0.1007666602+0.006785231676 L_{s}\right) z^{88} \\
& +\left(0.09895320635+0.006623533114 L_{s}\right) z^{89}+(0.09715805042 \\
& \left.+0.006491319205 L_{s}\right) z^{90}+\left(0.09544774254+0.006339815263 L_{s}\right) z^{91} \\
& +\left(0.09375349018+0.006216090979 L_{s}\right) z^{92}+(0.09213842100 \\
& \left.+0.006073938819 L_{s}\right) z^{93}+\left(0.09053742317+0.005957996834 L_{s}\right) z^{94} \\
& +\left(0.08901044095+0.005824439116 L_{s}\right) z^{95}+(0.08749577159 \\
& \left.+0.005715644039 L_{s}\right) z^{96}+\left(0.08605039426+0.005589998712 L_{s}\right) z^{97} \\
& +\left(0.08461576903+0.005487778501 L_{s}\right) z^{98}+(0.08324610956 \\
& \left.+0.005369429986 L_{s}\right) z^{99}+\left(0.08188581505+0.005273268529 L_{s}\right) z^{100} \\
& +0
\end{aligned}
$$

In order to present the high-order LME in a space-saving way, we refrain from showing the analytical result here and refer to HaaNiggetiedt.m attached to this paper for the exact expansion. After conversion to a quark-mass renormalised in $\overline{\mathrm{MS}}$ scheme, we find full agreement with ref. [15] up to $\mathcal{O}\left(z^{20}\right)$. 


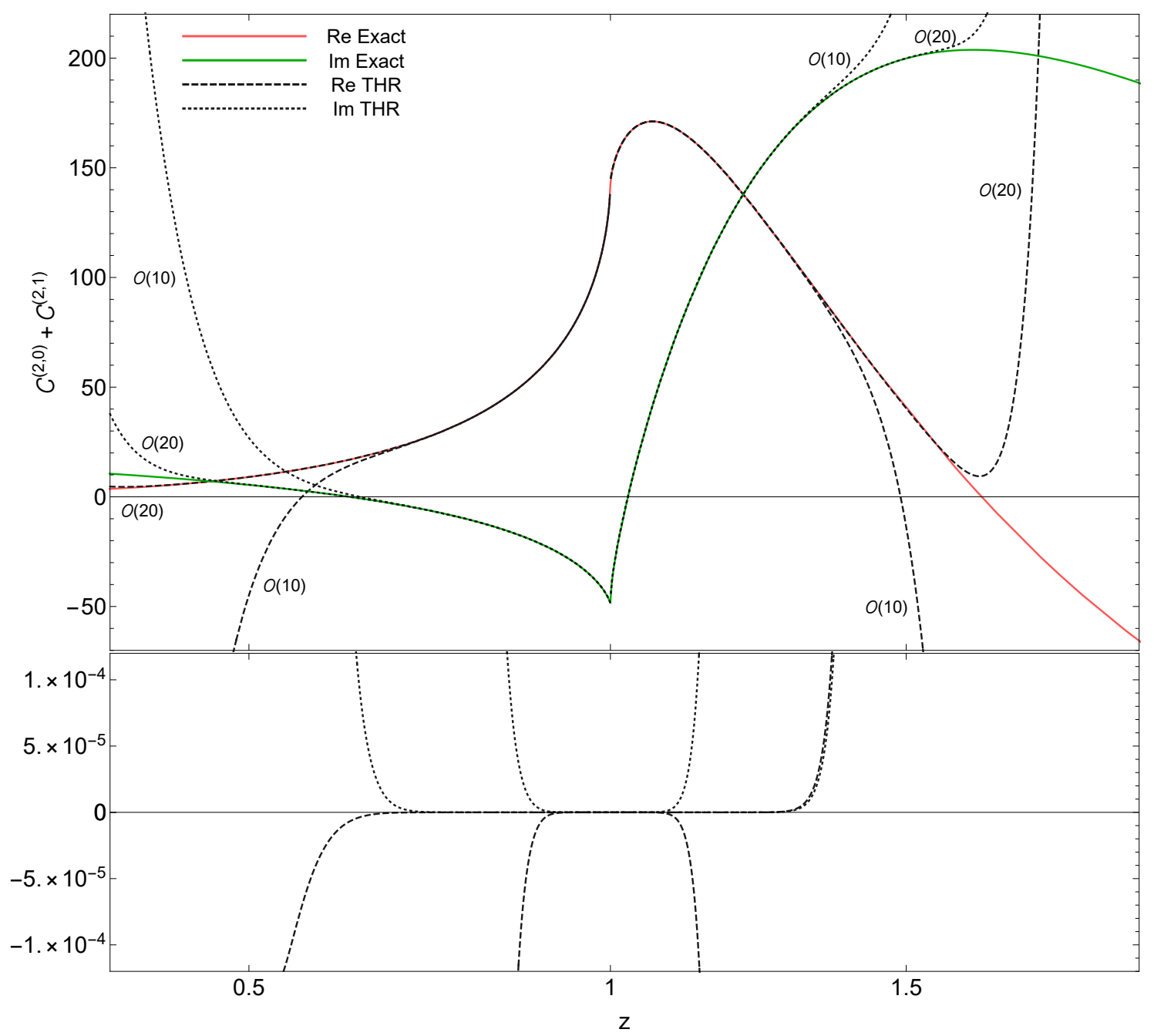

Figure 4. Comparison of the threshold expansion (THR) up to $\mathcal{O}\left((1-z)^{10}\right)$ and $\mathcal{O}\left((1-z)^{20}\right)$ with the sum $\mathcal{C}^{(2,0)}+\mathcal{C}^{(2,1)}$ evaluated numerically $\left(L_{\mu}=0\right)$. The absolute difference between the exact result and the expansions is shown in the bottom panel.

\section{B Threshold expansion}

$$
\begin{aligned}
C^{(2,0)}+C^{(2,1)}= & \sum_{n=0}^{\infty}\left(b_{n, 0}+b_{n, 1} L_{t}+b_{n, 2} L_{t}^{2}\right) t^{n}, \\
L_{t} \equiv & \ln (1-z), \quad t \equiv \sqrt{1-z}=\exp \left(L_{t} / 2\right), \\
\mathcal{C}^{(2,0)}+\mathcal{C}^{(2,1)}= & 143.7364241-48.71649342 i-177.3504510 t+(197.6561763 \\
& \left.-204.1570374 i+(148.1633930-170.5345217 i) L_{t}-27.14141210 L_{t}^{2}\right) t^{2} \\
& +\left(53.77375045+59.37899426 i-172.2570927 L_{t}\right) t^{3}+(-2182.218552 \\
& \left.+696.9359899 i+(70.58782969-113.6896812 i) L_{t}-72.92541030 L_{t}^{2}\right) t^{4}
\end{aligned}
$$




$$
\begin{aligned}
& +\left(2592.396897-775.0446867 i+(-940.1458201+289.5083958 i) L_{t}\right. \\
& \left.+92.15338451 L_{t}^{2}\right) t^{5}+(-6442.015967+2189.501187 i+(-156.2174980 \\
& \left.+79.58277681 i) L_{t}-56.78677940 L_{t}^{2}\right) t^{6}+(7101.227732-2217.403057 i \\
& \left.+(-2281.118997+800.9732283 i) L_{t}+314.5316023 L_{t}^{2}\right) t^{7} \\
& +\left(-12280.42300+4176.216119 i+(-416.8534109+376.8000861 i) L_{t}\right. \\
& \left.+38.55934333 L_{t}^{2}\right) t^{8}+(13282.39833-4186.062962 i+(-4084.925834 \\
& \left.+1495.448725 i) L_{t}+676.5815942 L_{t}^{2}\right) t^{9}+(-19513.93758 \\
& +6637.073703 i+(-634.2474597+760.6381049 i) L_{t} \\
& \left.+219.9642388 L_{t}^{2}\right) t^{10}+(20956.86608-6661.423781 i+(-6245.914043 \\
& \left.+2348.223277 i) L_{t}+1181.316740 L_{t}^{2}\right) t^{11}+(-28019.96998 \\
& +9579.038585 i+(-751.3909196+1220.072379 i) L_{t} \\
& \left.+490.9776099 L_{t}^{2}\right) t^{12}+(30014.75264-9648.580578 i+(-8657.469901 \\
& \left.+3342.107976 i) L_{t}+1829.712389 L_{t}^{2}\right) t^{13}+(-37710.95435 \\
& +13020.79214 i+(-722.4485167+1747.349447 i) L_{t} \\
& \left.+853.7398177 L_{t}^{2}\right) t^{14}+(40390.07403-13164.14293 i+(-11201.33327 \\
& \left.+4464.349253 i) L_{t}+2621.968191 L_{t}^{2}\right) t^{15}+(-48521.24097 \\
& +16986.69106 i+(-508.4575273+2336.654212 i) L_{t} \\
& \left.+1309.673614 L_{t}^{2}\right) t^{16}+(52049.17759-17230.58951 i+(-13736.34303 \\
& \left.+5705.036736 i) L_{t}+3557.958838 L_{t}^{2}\right) t^{17}+(-60399.79929 \\
& +21503.96892 i+(-75.04397778+2983.425813 i) L_{t} \\
& \left.+1859.791012 L_{t}^{2}\right) t^{18}+(64986.27918-21873.52288 i+(-16085.54882 \\
& \left.+7056.200309 i) L_{t}+4637.418784 L_{t}^{2}\right) t^{19}+(-73305.84110 \\
& \left.+26601.30209 i+(608.8980624+3683.967554 i) L_{t}+2504.848471 L_{t}^{2}\right) t^{20} \\
& +\left(79223.34316-27120.21267 i+(-18020.18143+8511.258872 i) L_{t}\right. \\
& \left.+5860.025054 L_{t}^{2}\right) t^{21}+(-87206.03702+32308.02280 i+(1572.025947 \\
& \left.+4435.206855 \text { i }) L_{t}+3245.433200 L_{t}^{2}\right) t^{22}+(94813.29712
\end{aligned}
$$


$-32998.77290 i+(-19238.78222+10064.66381 i) L_{t}$

$\left.+7225.436525 L_{t}^{2}\right) t^{23}+(-102072.6614+38653.66682 i+(2841.087074$

$\left.+5234.538704 i) L_{t}+4082.014739 L_{t}^{2}\right) t^{24}+(111846.4424$

$-39537.67660 i+(-19339.41674+11711.65750 i) L_{t}$

$\left.+8733.313071 L_{t}^{2}\right) t^{25}+(-117882.3077+45667.70560 i+(4441.290158$

$\left.+6079.718781 i) L_{t}+5014.977594 L_{t}^{2}\right) t^{26}+(130460.4625$

$-46765.46043 i+(-17782.29142+13448.10353 i) L_{t}$

$\left.+10383.32486 L_{t}^{2}\right) t^{27}+(-134614.9707+53379.38527 i+(6396.571505$

$\left.+6968.787760 i) L_{t}+6044.642843 L_{t}^{2}\right) t^{28}+(150854.8781$

$-54710.54167 i+(-13839.21226+15270.36352 i) L_{t}$

$\left.+12175.15662 L_{t}^{2}\right) t^{29}+(-152253.3725+61817.62989 i+(8729.792470$

$\left.+7900.016030 i) L_{t}+7171.282997 L_{t}^{2}\right) t^{30}+(173311.2659$

$-63401.10391 i+(-6525.100034+17175.20536 i) L_{t}$

$\left.+14108.50932 L_{t}^{2}\right) t^{31}+(-170782.4563+71010.98452 i+(11462.89010$

$\left.+8871.862283 i) L_{t}+8395.132546 L_{t}^{2}\right) t^{32}+(198221.1192$

$\left.-72865.02611 i+(5494.900238+19159.73344 i) L_{t}+16183.10042 L_{t}^{2}\right) t^{33}$

$+\left(-190188.9992+80987.58341 i+(14616.99489+9882.941815 i) L_{t}\right.$

$\left.+9716.395645 L_{t}^{2}\right) t^{34}+(226123.9546-83129.83963 i+(24031.45904$

$\left.+21221.33438 i) L_{t}+18398.66346 L_{t}^{2}\right) t^{35}+(-210461.3098$

$\left.+91775.13446 i+(18212.52478+10932.00180 i) L_{t}+11135.25185 L_{t}^{2}\right) t^{36}$

$+\left(257759.2233-94222.70360 i+(51542.09097+23357.63429 i) L_{t}\right.$

$\left.+20754.94726 L_{t}^{2}\right) t^{37}+(-231588.9897+103400.9142 i+(22269.26158$

+12017.90168 i) $\left.L_{t}+12651.86050 L_{t}^{2}\right) t^{38}+(294136.8713$

$\left.-106170.3924 i+(91363.20676+25566.46448 i) L_{t}+23251.71496 L_{t}^{2}\right) t^{39}$

$+\left(-253562.7417+115891.7695 i+(26806.41403+13139.59741 i) L_{t}\right.$

$\left.+14266.36404 L_{t}^{2}\right) t^{40}+\mathcal{O}\left(t^{41}\right)$. 


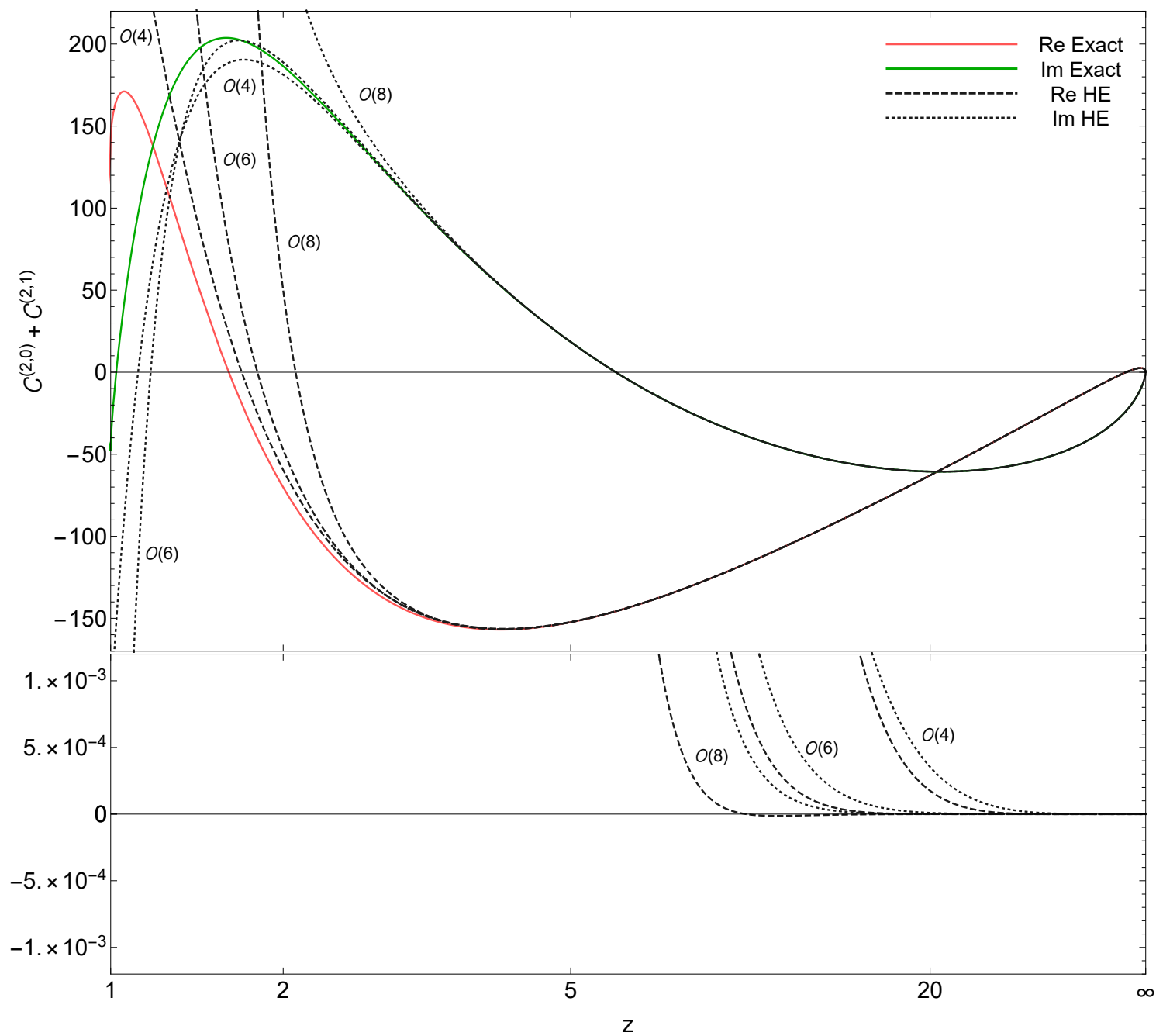

Figure 5. Comparison of the high-energy expansion (HE) up to $\mathcal{O}\left(1 / z^{4}\right), \mathcal{O}\left(1 / z^{6}\right)$ and $\mathcal{O}\left(1 / z^{8}\right)$ with the sum $\mathcal{C}^{(2,0)}+\mathcal{C}^{(2,1)}$ evaluated numerically $\left(L_{\mu}=0\right)$. The absolute difference between the exact result and the expansions is shown in the bottom panel.

\section{High-energy expansion}

$$
\begin{aligned}
C^{(2,0)}+C^{(2,1)}= & \sum_{n=1}^{\infty} \sum_{k=0}^{6} c_{n, k} L_{s}^{k} z^{-n}, \quad L_{s} \equiv \ln \left(-\frac{s}{M^{2}}-i 0^{+}\right) \\
\mathcal{C}^{(2,0)}+\mathcal{C}^{(2,1)}= & \left(54.56087661-105.9626626 L_{s}+8.887259013 L_{s}^{2}+6.645715659 L_{s}^{3}\right. \\
& \left.+0.8289545430 L_{s}^{4}+0.03333333333 L_{s}^{5}-0.001851851852 L_{s}^{6}\right) z^{-1} \\
& +\left(85.66611966+12.14331252 L_{s}-34.87431988 L_{s}^{2}-11.29224411 L_{s}^{3}\right. \\
& \left.-0.8617012615 L_{s}^{4}+0.01718750000 L_{s}^{5}+0.001099537037 L_{s}^{6}\right) z^{-2} \\
& +\left(4.242650309+54.17459631 L_{s}+4.950031592 L_{s}^{2}+1.341075352 L_{s}^{3}\right.
\end{aligned}
$$




$$
\begin{aligned}
& \left.-0.9050441963 L_{s}^{4}-0.02955729167 L_{s}^{5}-0.001410590278 L_{s}^{6}\right) z^{-3} \\
& +\left(-99.58994875+39.36666234 L_{s}+16.69670684 L_{s}^{2}-2.994924396 L_{s}^{3}\right. \\
& \left.-0.4321117491 L_{s}^{4}+0.07813946759 L_{s}^{5}-0.002267795139 L_{s}^{6}\right) z^{-4} \\
& +\left(-13.01069569-30.23605994 L_{s}-33.09478959 L_{s}^{2}+15.42454676 L_{s}^{3}\right. \\
& \left.-0.1114137280 L_{s}^{4}-0.08451605903 L_{s}^{5}-0.004180230035 L_{s}^{6}\right) z^{-5} \\
& +\left(-50.77508814+315.6787714 L_{s}-32.49111907 L_{s}^{2}-27.01662191 L_{s}^{3}\right. \\
& \left.+0.2417871470 L_{s}^{4}+0.2111745877 L_{s}^{5}+0.004659921152 L_{s}^{6}\right) z^{-6} \\
& +\left(-220.1240019-1889.592657 L_{s}+543.4130382 L_{s}^{2}+67.78095852 L_{s}^{3}\right. \\
& \left.-9.594792355 L_{s}^{4}-0.05716437042 L_{s}^{5}-0.02886098226 L_{s}^{6}\right) z^{-7} \\
& +\left(488.0690355+11655.54167 L_{s}-4220.431798 L_{s}^{2}-99.26817518 L_{s}^{3}\right. \\
& \left.+67.30519611 L_{s}^{4}-2.224095015 L_{s}^{5}+0.08744108412 L_{s}^{6}\right) z^{-8}+\mathcal{O}\left(z^{-9}\right) .
\end{aligned}
$$

The parts of the numerical coefficients of terms proportional to $L_{s}^{k} / z$ for $k \in\{6,5,4,3\}$, which stem from $\mathcal{C}^{(2,0)}$, comply with the exact coefficients predicted recently in refs. [19, 20].

\section{Supplemental material}

The supplemental material, HaaNiggetiedt.m attached to this paper, in form of a single file can be imported in Wolfram Mathematica for subsequent analysis. All variables are explained in the header of this file. The main function returns the form factor as a series in aspi $\equiv \alpha_{s} / \pi$ :

CHaa $[z, \mathrm{nh}, \mathrm{nl}, \mathrm{QQsum}, \mathrm{Lmu}]-\mathcal{C}$, eq. (2.3);

The function CHaa $[z, \mathrm{nh}, \mathrm{nl}, \mathrm{QQsum}, \mathrm{Lmu}]$ is entirely based on the aforementioned expansions and interpolations. Hence, the analytical results of ref. [16] are not exploited. For the benefit of the reader, we provide all constituents of the form factor in terms of expansions and interpolation tables. The following functions are evaluated at $L_{\mu}=0$ :

C0 [z], C1 [z], C2 [z, nh, nl, QQsum $]-\mathcal{C}^{(0)}, \mathcal{C}^{(1)}$ and $\mathcal{C}^{(2)}$, eqs. (2.3) and (2.4);

C2LMEn00 [z], C2LMEnh1[z], C2LMEn11[z], C2LMEQQsum $[z]$

- large-mass expansion of $\mathcal{C}^{(2,0)}, \mathcal{C}^{(2,1)}$ (appendix A), $\mathcal{C}^{(2,2)}$ and $\mathcal{C}^{(2,3)}$;

C2THRn00[z], C2THRnh1[z], C2THRn11[z], C2THRQQsum[z]

- threshold expansion of $\mathcal{C}^{(2,0)}, \mathcal{C}^{(2,1)}$ (appendix B), $\mathcal{C}^{(2,2)}$ and $\mathcal{C}^{(2,3)}$;

C2HEn00 [z], C2HEnh1 $[z]$, C2HEnl1 $[z]$, C2HEQQsum $[z]$

- high-energy expansion of $\mathcal{C}^{(2,0)}, \mathcal{C}^{(2,1)}$ (appendix $\mathrm{C}$ ), $\mathcal{C}^{(2,2)}$ and $\mathcal{C}^{(2,3)}$;

C2TABn00[z], C2TABnh1[z], C2TABnl1[z], C2TABQQsum $[z]$

- interpolation of $\mathcal{C}^{(2,0)}, \mathcal{C}^{(2,1)}$ (tables 1 and 2), $\mathcal{C}^{(2,2)}$ and $\mathcal{C}^{(2,3)}$. 
One must supply numerical values for $z$. The large-mass expansion of $\mathcal{C}^{(2)}\left[L_{\mu}=0\right]$ with exact coefficients can be called with C2LME. Its dependence on $n_{h}, n_{l}$ and the sum over electric charges is kept variable.

All functions are evaluated with fixed gauge group constants $C_{A}=3, C_{F}=4 / 3$, $T_{F}=1 / 2$.

Open Access. This article is distributed under the terms of the Creative Commons Attribution License (CC-BY 4.0), which permits any use, distribution and reproduction in any medium, provided the original author(s) and source are credited.

\section{References}

[1] M.L. Czakon and M. Niggetiedt, Exact quark-mass dependence of the Higgs-gluon form factor at three loops in QCD, JHEP 05 (2020) 149 [arXiv:2001.03008] [INSPIRE].

[2] ATLAS collaboration, Observation of a new particle in the search for the Standard Model Higgs boson with the ATLAS detector at the LHC, Phys. Lett. B 716 (2012) 1 [arXiv: 1207.7214] [INSPIRE].

[3] CMS collaboration, Observation of a New Boson at a Mass of $125 \mathrm{GeV}$ with the CMS Experiment at the LHC, Phys. Lett. B 716 (2012) 30 [arXiv:1207.7235] [INSPIRE].

[4] LHC Higgs Cross Section Working Group, Handbook of LHC Higgs Cross Sections: 4. Deciphering the Nature of the Higgs Sector, arXiv:1610.07922 [INSPIRE].

[5] H.-Q. Zheng and D.-D. Wu, First order QCD corrections to the decay of the Higgs boson into two photons, Phys. Rev. D 42 (1990) 3760 [InSPIRE].

[6] S. Dawson and R.P. Kauffman, QCD corrections to $H \rightarrow \gamma \gamma$, Phys. Rev. D 47 (1993) 1264 [INSPIRE].

[7] A. Djouadi, M. Spira, J.J. van der Bij and P.M. Zerwas, QCD corrections to $\gamma \gamma$ decays of Higgs particles in the intermediate mass range, Phys. Lett. B 257 (1991) 187 [INSPIRE].

[8] K. Melnikov and O.I. Yakovlev, Higgs $\rightarrow$ two photon decay: QCD radiative correction, Phys. Lett. B 312 (1993) 179 [hep-ph/9302281] [INSPIRE].

[9] A. Djouadi, M. Spira and P.M. Zerwas, Two photon decay widths of Higgs particles, Phys. Lett. B 311 (1993) 255 [hep-ph/9305335] [INSPIRE].

[10] M. Spira, A. Djouadi, D. Graudenz and P.M. Zerwas, Higgs boson production at the LHC, Nucl. Phys. B 453 (1995) 17 [hep-ph/9504378] [inSPIRE].

[11] J. Fleischer, O.V. Tarasov and V.O. Tarasov, Analytical result for the two loop QCD correction to the decay $H \rightarrow \gamma$, Phys. Lett. B 584 (2004) 294 [hep-ph/0401090] [InSPIRE].

[12] R. Harlander and P. Kant, Higgs production and decay: Analytic results at next-to-leading order QCD, JHEP 12 (2005) 015 [hep-ph/0509189] [INSPIRE].

[13] U. Aglietti, R. Bonciani, G. Degrassi and A. Vicini, Analytic Results for Virtual QCD Corrections to Higgs Production and Decay, JHEP 01 (2007) 021 [hep-ph/0611266] [INSPIRE].

[14] M. Steinhauser, Corrections of $O\left(\alpha_{s}^{2}\right)$ to the decay of an intermediate mass Higgs boson into two photons, in The Higgs puzzle - what can we learn from LEP-2, LHC, NLC and FMC?, proceedings of the Ringberg Workshop, Tegernsee, Germany, 8-13 December 1996, pp. 177-185 [hep-ph/9612395] [INSPIRE]. 
[15] P. Maierhöfer and P. Marquard, Complete three-loop QCD corrections to the decay $H \rightarrow \gamma \gamma$, Phys. Lett. B 721 (2013) 131 [arXiv:1212.6233] [InSPIRE].

[16] R.V. Harlander, M. Prausa and J. Usovitsch, The light-fermion contribution to the exact Higgs-gluon form factor in QCD, JHEP 10 (2019) 148 [Erratum JHEP 08 (2020) 101] [arXiv: 1907.06957] [INSPIRE].

[17] R. Akhoury, H. Wang and O.I. Yakovlev, On the Resummation of large QCD logarithms in $H \rightarrow \gamma \gamma$ decay, Phys. Rev. D 64 (2001) 113008 [hep-ph/0102105] [INSPIRE].

[18] C. Anastasiou and A. Penin, Light Quark Mediated Higgs Boson Threshold Production in the Next-to-Leading Logarithmic Approximation, JHEP 07 (2020) 195 [Erratum JHEP 01 (2021) 164] [arXiv: 2004.03602] [INSPIRE].

[19] Z.L. Liu, B. Mecaj, M. Neubert and X. Wang, Factorization at Subleading Power and Endpoint Divergences in Soft-Collinear Effective Theory, arXiv:2009.04456 [INSPIRE].

[20] Z.L. Liu, B. Mecaj, M. Neubert and X. Wang, Factorization at subleading power and endpoint divergences in $h \rightarrow \gamma \gamma$ decay. Part II. Renormalization and scale evolution, JHEP 01 (2021) 077 [arXiv: 2009.06779] [INSPIRE].

[21] K. Melnikov and T. van Ritbergen, The Three loop relation between the MS-bar and the pole quark masses, Phys. Lett. B 482 (2000) 99 [hep-ph/9912391] [INSPIRE].

[22] K. Melnikov and T. van Ritbergen, The Three loop on-shell renormalization of QCD and QED, Nucl. Phys. B 591 (2000) 515 [hep-ph/0005131] [INSPIRE].

[23] A. Mitov and S. Moch, The Singular behavior of massive QCD amplitudes, JHEP 05 (2007) 001 [hep-ph/0612149] [INSPIRE].

[24] N. Gray, D.J. Broadhurst, W. Grafe and K. Schilcher, Three Loop Relation of Quark (Modified) Ms and Pole Masses, Z. Phys. C 48 (1990) 673 [InSPIRE].

[25] W. Bernreuther and W. Wetzel, Decoupling of Heavy Quarks in the Minimal Subtraction Scheme, Nucl. Phys. B 197 (1982) 228 [Erratum ibid. 513 (1998) 758] [inSPIRE]. 